\title{
Congenital renal tubular dysplasia and skull ossification defects similar to teratogenic effects of angiotensin converting enzyme (ACE) inhibitors
}

\author{
Dhavendra Kumar, Gail Moss, Rob Primhak, Robert Coombs
}

Centre for Human Genetics, 117

Manchester Road, Sheffield S10 5DN, UK D Kumar

\section{Department of \\ Paediatrics, Sheffield Children's Hospital, Sheffield, UK \\ G Moss \\ R Primhak}

Neonatal Unit, Jessop Hospital for Women, Sheffield, UK

$\mathrm{R}$ Coombs

Correspondence to: Dr Kumar.

Received 4 March 1996 Revised version accepted for publication 6 March 1997

\begin{abstract}
An apparently autosomal recessive syndrome of congenital renal tubular dysplasia and skull ossification defects is described in five infants from two separate, consanguineous, Pakistani Muslim kindreds. The clinical, pathological, and radiological features are similar to the phenotype associated with fetal exposure to angiotensin converting enzyme (ACE) inhibitors: intrauterine growth retardation, skull ossification defects, and fetal/ neonatal anuric renal failure associated with renal tubular dysplasia. There was no fetal exposure to ACE inhibitors in the affected infants. Phenotypic similarities between these familial cases and those associated with ACE inhibition suggest an abnormality of the "renin-angiotensinaldosterone" system (RAS). It is postulated that the molecular pathology in this uncommon autosomal recessive proximal renal tubular dysgenesis could be related to mutations of the gene systems governing the RAS.

(F Med Genet 1997;34:541-545)
\end{abstract}

Family 1

I

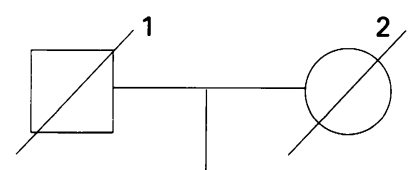

II

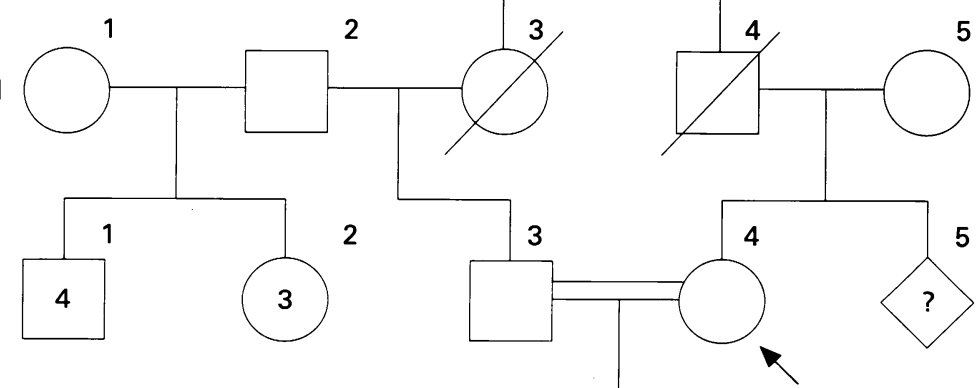

IV

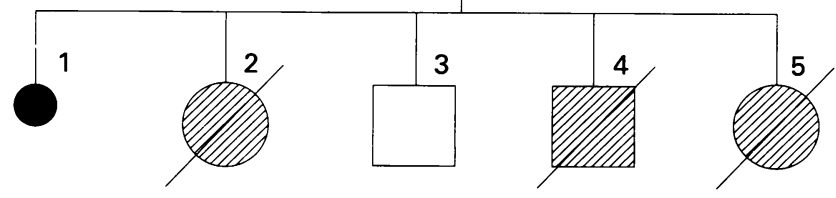

Figure 1 Pedigree of family 1.

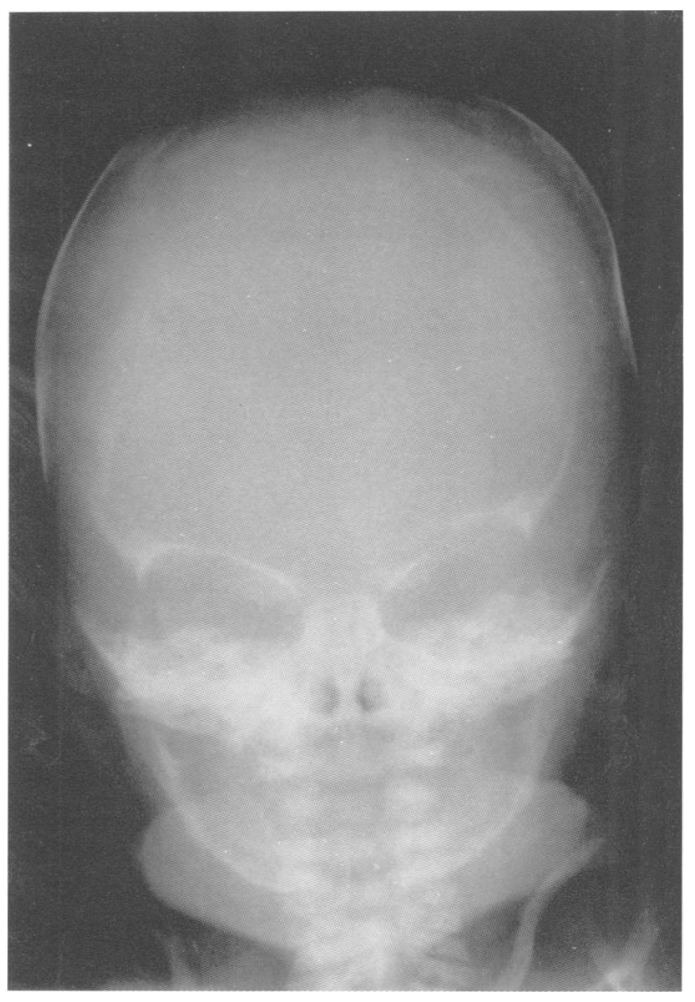

Figure 2 Skull radiograph of IV.5, family 1: note poor ossification and wide skull sutures.

Keywords: proximal renal tubular dysplasia; reninangiotensin system (RAS); angiotensin converting enzyme (ACE) inhibitors; fetal ACE inhibitors syndrome

Several reports of primary renal tubular dysplasia (RTD) ${ }^{1}$ have been published since the original description. ${ }^{2}$ Recurrence in sibs ${ }^{34}$ and parental consanguinity ${ }^{5}$ favour autosomal recessive inheritance (MIM 267430). ${ }^{1}$ The precise molecular mechanism leading to the phenotype of RTD is not clear. The putative gene(s) is yet to be mapped and cloned.

This report describes five infants from two unrelated consanguineous Muslim families presenting with multiple congenital anomalies (MCA) including RTD. The purposes of this report are to delineate further the phenotype of autosomal recessive RTD and to draw attention to striking similarities with the teratogenic effects of maternal administration of angiotensin converting enzyme (ACE) inhibitors (for example, captopril, enalapril), which include skull ossification defects and anuric neonatal renal failure. ${ }^{67}$ The phenotype of "fetal ACE inhibitors syndrome" is now 


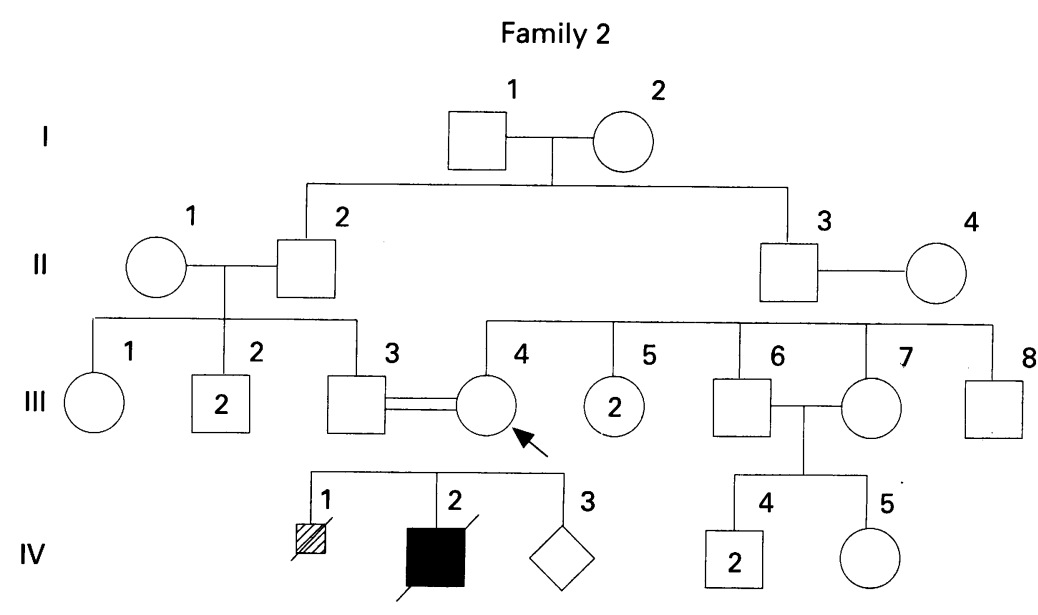

Figure 3 Pedigree of family 2.
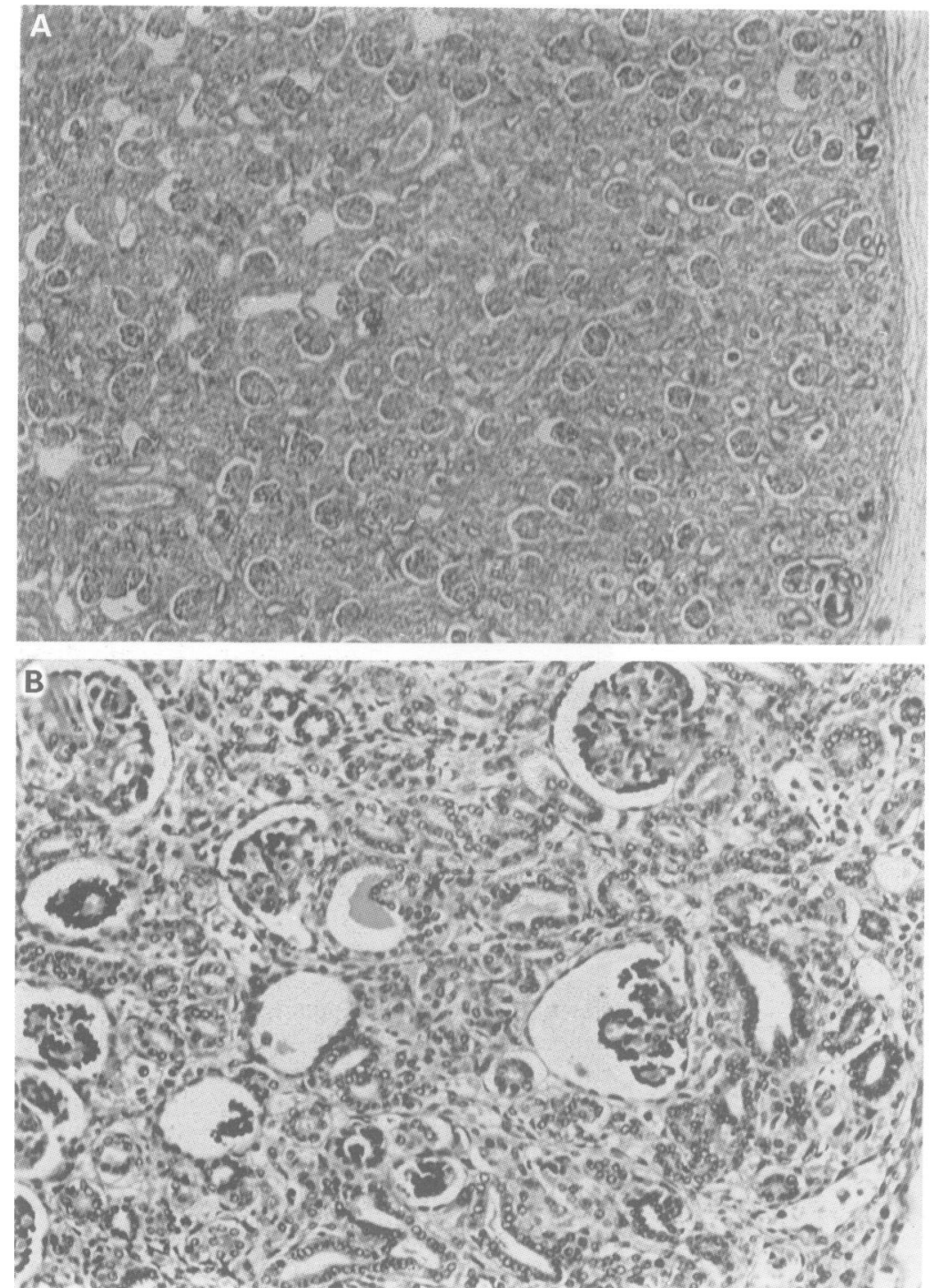

Figure 4 Renal histopathological changes in IV.2, family 2 showing marked deficiency and dysgenesis of proximal renal tubules; adjoining glomeruli are not well formed and appear crowded, (A) under low magnification, (B) under high magnification.

accepted. ${ }^{8}$ We believe that this is the first report illustrating similarities between the phenotypes associated with fetal ACE exposure and genetically determined RTD. We believe that the molecular pathology of the RAS might be associated with the distinct and recognisable syndrome of primary renal tubular dysplasia with skull ossification defects.

\section{Case reports}

FAMILY 1

A Pakistani Muslim woman (III.4, fig 1) presented in her second pregnancy (IV.2) complicated with oligohydramnios. Parental consanguinity was noted at the time of antenatal booking. The first pregnancy (IV.1) had miscarried spontaneously at around 12 weeks' gestation. A female infant was delivered at term who looked dysmorphic (abnormally shaped, small head and joint contractures). A clinical diagnosis of Potter syndrome was made, but renal ultrasound scan showed that both kidneys were of normal size, structure, and echogenicity. She died within 24 hours of birth; consent for necropsy was not given. The next conception produced a healthy male infant (IV.3).

The fourth pregnancy resulted in a live born male infant (IV.4). Dysmorphic features noticed at birth included Potter facies, abnormally shaped, small head, and large joint contractures. Skull $x$ ray showed poor ossification of the skull vault. The baby was anuric from birth and renal failure associated with severe hypotension persisted until death at 7 weeks. Renal ultrasound examination indicated apparently normal kidneys. Permission for necropsy was again refused. The last pregnancy produced a female infant (IV.5). Oligohydramnios was suspected in the later part of pregnancy; a fetal ultrasound examination showed normal kidneys. At birth the baby had an abnormally shaped skull with widely separated sutures (fig 2) and large joint contractures. The baby was anuric from birth with severe hypotension. An ultrasound examination showed normal sized kidneys with normal echogenicity. Biochemical investigations indicated high plasma renin activity (PRA). This baby died from respiratory failure within a few hours of birth and consent for necropsy was again refused.

FAMILY 2

A consanguineous Pakistani Muslim couple (III.3, III.4, fig 3) presented in their second pregnancy. The first pregnancy terminated prematurely in a male stillbirth with Potter syndrome at 29 weeks' gestation (IV.1). The kidneys could not be visualised on antenatal ultrasound scan. Consent for necropsy was not given. The product of the second pregnancy, a live born male infant delivered at term (IV.2), had dysmorphic features consistent with the Potter phenotype. In addition, the head looked abnormal with widely separated sutures and a large anterior fontanelle. Intensive care was required in view of severe respiratory distress probably related to pulmonary hypoplasia, profound hypotension, and anuric renal failure. Both kidneys appeared normal in shape and size with normal echogenicity on renal ultrasound examinations. Despite intensive management including peritoneal dialysis, the baby died at 17 days of age.

Biochemical investigations showed high PRA and low angiotensin I levels. Molecular genetic investigations showed the child to be a 


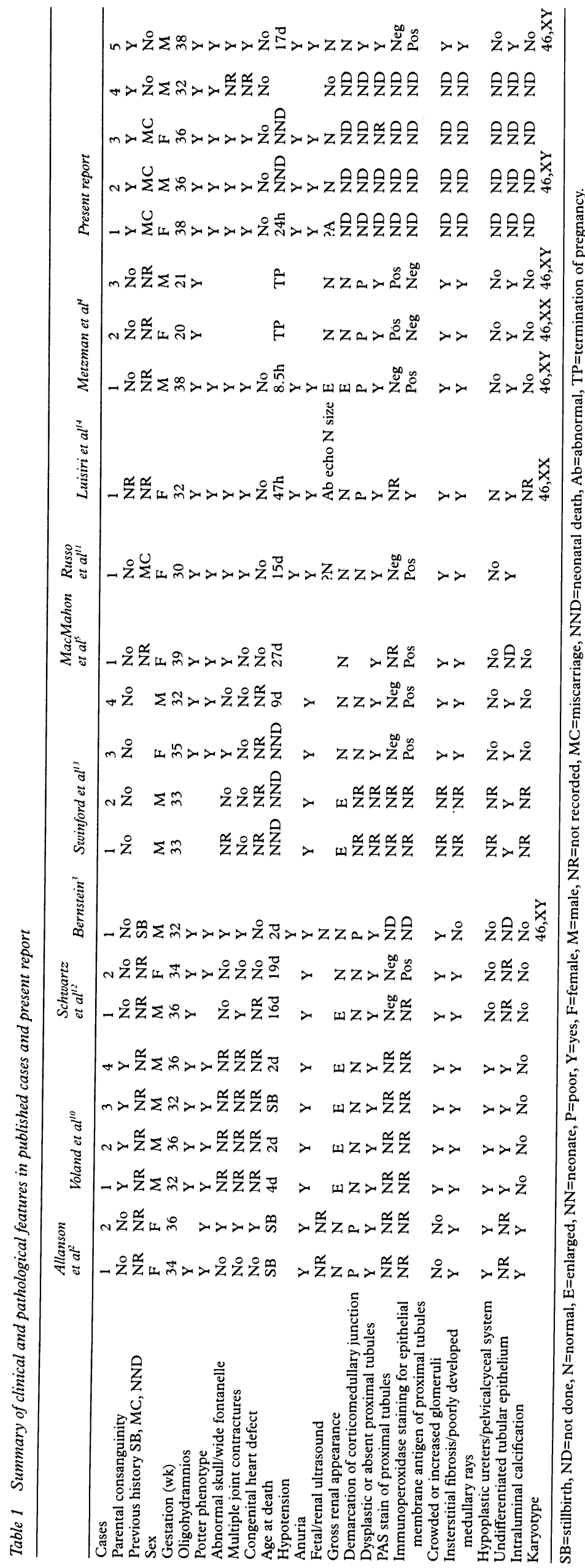

homozygote for the insertion polymorphism (II) in the ACE gene. Blood was not available for determination of parental ACE genotype or molecular analysis of the angiotensinogen gene in either the parents or infant.

Necropsy showed macroscopic pulmonary hypoplasia, microscopic evidence of pulmonary immaturity, and a cuboidal alveolar epithelium. Interstitial emphysema and lobular overdistension were also present, consistent with changes secondary to mechanical ventilation. Histopathological examination of the kidneys showed variably sized glomerular cysts, very poor maturation of cortical tubules, and nodular collections of tubules suggestive of early tubular dysplasia (fig 4A, B). There was no evidence of acute tubular necrosis. These findings prompted the diagnosis of "proximal renal tubular dysgenesis".

The third pregnancy resulted in a healthy male infant (IV.3) who had a normal skull $x$ ray and renal ultrasound scan.

\section{Discussion}

There are two aspects to this report: firstly, further delineation of the syndrome of autosomal recessive proximal RTD and, secondly, examination of the evidence provided by the clinical resemblance between this and the "fetal ACE inhibitor syndrome".

These reported families are Pakistani Muslim with parental consanguinity. The affected pregnancies resulted in early loss, stillbirth, or infants presenting after second trimester oligohydramnios with intrauterine growth retardation, skull ossification defects, and early onset renal failure. All affected liveborn infants died in the neonatal period. It is postulated that the same aetiology could account for all these presentations.

The clinical picture is compatible with Potter syndrome or the oligohydramnios sequence. ${ }^{9}$ This is usually associated with chronic leakage of amniotic fluid from midgestation, severe bilateral renal malformation including agenesis, dysgenesis, polycystic kidney disease, and multicystic kidney disease, or critical urinary tract obstruction. However, apart from infant IV.1 (family 2) where bilateral agenesis was suspected on antenatal ultrasound scan alone, no evidence of these underlying problems was found in the affected pregnancies.

Profound hypotension resistant to treatment was a major feature in infants IV.4 (family 1) and IV.2 (family 2) which could account for the associated renal failure. However, in this circumstance features of acute tubular necrosis (ATN) might be expected on histology. In contrast, where renal histopathology was available in infant IV.2 (family 2), marked glomerular proliferation and poor differentiation were shown and were considered to be compatible with proximal renal tubular dysgenesis. These findings are similar to those seen in autosomal recessive proximal RTD (MIM 267430).'

Metzman $e^{2} \mathrm{al}^{4}$ reviewed the previous reports of inherited proximal RTD, which is now recognised as a specific phenotype comprising proximal RTD as the major anomaly with occasional occurrence of ossification defects of 
Renin Angiotensin System

Renin secretion from the juxta glomerular apparatus

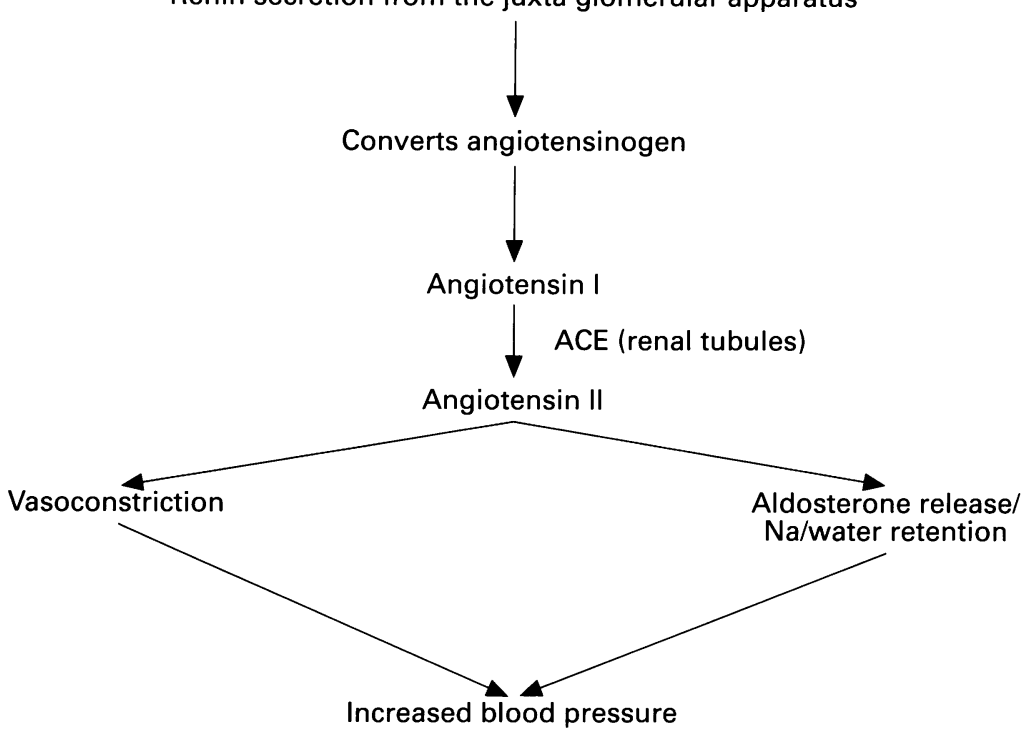

Figure 5 The pathway of the renin-angiotensin system (RAS).

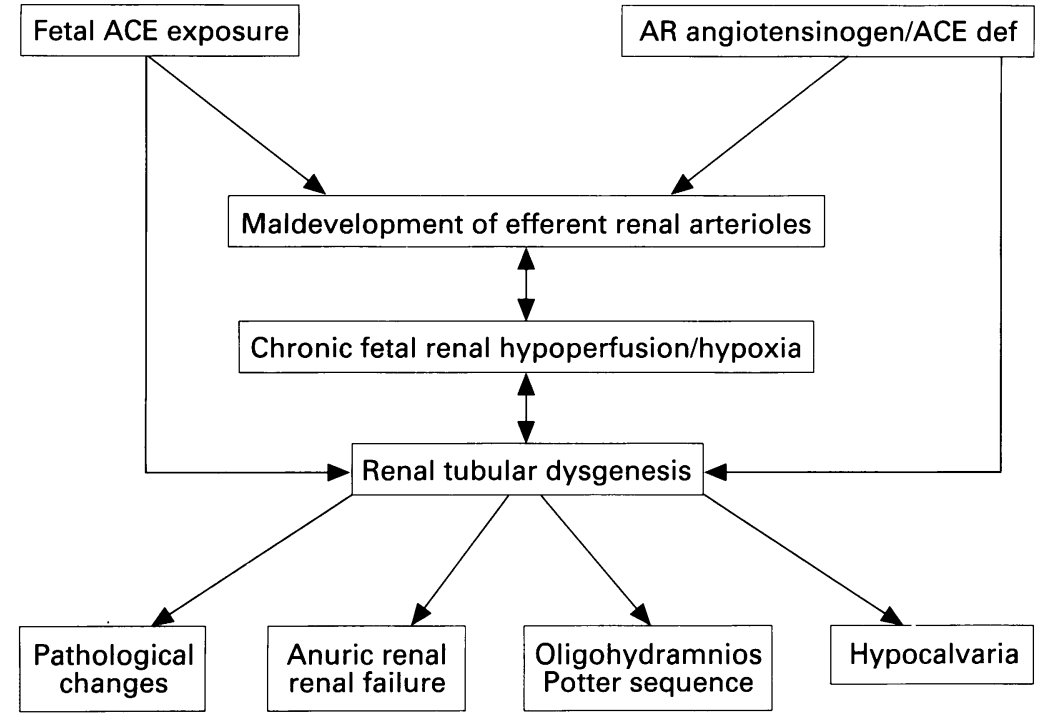

Figure 6 Pathological events in fetal ACE inhibitor exposure and autosomal recessive proximal RTD leading to development of the similar clinical phenotype.

the skull. Table 1 summarises the phenotypic findings in our two families together with those of previously published cases. ${ }^{25}{ }^{10-14}$ We believe that the infants in this report are further cases of autosomal recessive proximal RTD manifesting with pre- or perinatal anuric renal failure and congenital ossification defects of the skull.

The mechanism of inherited proximal RTD and its associated findings have not been elucidated. In our cases, investigations in infants IV.4 (family 1 ) and IV.2 (family 2) showed evidence of raised renin activity in both, together with a low level of angiotensin I (Ang I) in infant IV.2 (family 2). Unfortunately, further investigation in the affected families was precluded by insufficient blood samples. The raised plasma renin activity suggests activation of the RAS as might be expected in sick, hypotensive neonates. However, a low Ang I would suggest a block in the RAS which may in turn be the primary cause of the profound hypotension. Possible causes of this combination of findings include a qualitative abnormality of the circulating renin or a quantitative or qualitative abnormality of angiotensinogen which may be related to an abnormality in the angiotensin gene system (1q42-q44). It is interesting to speculate how these or the consequent abnormalities further down the pathway of the RAS could generate the clinical findings (fig 5). Consideration of the fetal ACE inhibitor syndrome may provide further insight.

There is striking similarity between the phenotype of inherited proximal RTD and the spectrum of developmental abnormalities resulting from probable teratogenic effects of ACE inhibitors. ${ }^{67}{ }^{75-18}$ Concerns about possible adverse fetal effects of ACE inhibitors were raised soon after their introduction as antihypertensive agents. ${ }^{19}{ }^{20}$ Fetal ACE inhibitor syndrome is now accepted as an entity whose major features are intrauterine growth retardation, skull ossification defects, and proximal renal tubular dysgenesis. ${ }^{8}$ There is an associated increased incidence of intrauterine deaths, stillbirths, and perinatal deaths which have been ascribed to oligohydramnios, fetal hypotension, and fetal/neonatal anuric renal failure. The latter has been considered secondary to decreased pressure and blood flow in the efferent glomerular arteriole secondary to decreased levels of angiotensin II (Ang II). This in turn is thought to contribute to the maldevelopment of the renal tubules (fig 6). The underlying mechanisms for the other associated findings are uncertain.

Despite their different chemical structures, all ACE inhibitors block the conversion of Ang I to Ang II with consequent fall in Ang II levels and other substances further down the RAS and rise in those proximal to the block (fig 5). We have speculated that our results might suggest a block in the RAS in inherited proximal RTD. If this is accepted, which of the biochemical abnormalities common to both of these situations could explain the clinical phenotype? Of the possibilities, recent work with Ang II suggests that this might be an important factor. ${ }^{21}$

Ang II is an octapeptide whose functions in vasoconstriction, regulation of glomerular filtration rate, and stimulation of aldosterone release are well known. More recently the growth promoting effects of Ang II on extrarenal tissues, in particular vascular smooth muscle cells, have been characterised. ${ }^{22}{ }^{23}$ However, it is now recognised that the RAS, and most notably Ang II, may have other more complex roles including growth regulation in renal tissue itself. ${ }^{21}$

In vitro studies have shown that Ang II induces hypertrophy of proximal tubular cells. ${ }^{21}$ Studies in the rat suggest a role for the RAS in early postnatal and maybe fetal nephronogenesis and growth. ${ }^{24}$ Furthermore, Ang II has been shown to induce transcription of some immediate early genes in a murine proximal tubular cell line ${ }^{25}{ }^{26}$ and also to stimulate expression of homeobox gene 2.3 in the same cell line. ${ }^{26}$ The clinical implications of these findings are unclear and none of these in 
vitro studies has investigated the effect of low RAS activity or Ang II levels. Nevertheless, it is interesting to hypothesise that this may contribute to proximal tubular dysgenesis via an effect on regulation of nephronal growth in addition to any changes in glomerular haemodynamics. The role of Ang II in the induction of early genes remains to be proven, but a lack of Ang II may conceivably contribute to the embryopathy seen in both inherited proximal RTD and the fetal ACE inhibitor syndrome.

We believe that this report shows the similarities between the uncommon genetic syndrome of autosomal recessive proximal RTD and the drug induced phenotype associated with fetal exposure to ACE inhibitors. Our limited investigations have not identified a specific gene abnormality in the former. Consideration of the available results together with knowledge of the fetal ACE inhibitor syndrome and functions of Ang II has led us to suggest that an abnormality in the RAS may be a fundamental problem in inherited proximal RTD. The angiotensinogen gene system may be a likely candidate gene, although others within the RAS may be responsible. More detailed investigation of the RAS in further cases is needed to establish whether this is true and to delineate the molecular pathology of the syndrome of autosomal recessive proximal RTD.

The authors of this report wish to acknowledge invaluable help and advice from Drs $\mathrm{C} \mathrm{J} \mathrm{H}$ Padfield and Dick Variend, consultant histopathologists, in Nottingham and Sheffield respectively. The renal histopathology in the affected boy in the second family was reported by Dr Jane Zuccollo of the histopathology department of the Queen's Medical Centre, Nottingham.

1 McKusick VA. Mendelian inheritance in man - catalog of autosomal and $X$-linked disorders. 11 th ed. Baltimore: Johns somal and X-linked disorders. 11 .
Hopkins University Press, 1993.

2 Allanson JE, Pantzar JT, Macleod PM. Possible new autosomal recessive syndrome with unusual histopathological changes. Am $\mathcal{f}$ Med Genet 1983;16:57-60.

3 Bernstein J. Renal tubular dysgenesis. Pediatr Pathol 1988;8: 453-56.

4 Metzman RA, Husson MA, Dellers EA. Renal tubular dysgenesis: a description of early renal maldevelopment in siblings. Pediatr Pathol 1993;13:239-48.
5 MacMahon P, Blackie RAS, House MJ, et al. A further family with congenital renal proximal tubular dysgenesis. $f \mathrm{Med}$ Genet 1990;27:395-8.

6 Cunnif C, Jones KL, Phillipson J, Benirschke K, Short S, Wujek J. Oligohydramnios sequence and renal malformation associated with maternal enalapril use. Am $\mathcal{f}$ Obstet Gynecol 1990;162:187-9.

7 Barr M Jr, Cohen MM Jr. ACE inhibitor fetopathy and hypocalvaria: the kidney-skull connection. Teratology 1991; 44:485-95.

8 Winter R, Baraitser M. The London Dysmorphology Database. Oxford: Oxford University Press, 1993.

9 Jones K. Smith's recognizable patterns of human malformation. 5th ed. Philadelphia: W B Saunders, 1995:572-3.

10 Voland JR, Hawkins EP, Wells TR, Sauders B, Jones M Benirschke K. Congenital hypernephronic nephronomegaly with tubular dysgenesis: a distinctive inherited renal anomaly. Pediatr Pathol 1985;4:231-45.

11 Russo R, D'Armiento $M$, Vecchione $R$. Renal tubular dysgenesis and very large cranial fontanels in a family with acrocephalosyndactyly SC type. Am f Med Genet 1991;39: $482-5$.

12 Schwartz BR, Lage JM, Pober BR, Driscoll SG. Isolated congenital renal tubular malformation in siblings. Hum Pathol 1985;17:231-45.

13 Swinford AE, Bernstein J, Toriella H, Higgins JV. Renal tubular dysgenesis: delayed onset of oligohydramnios. $A m^{\mathcal{F}}$ Med Genet 1989;32:127-32.

14 Luisiri A, Salinas-Madrigal L, Noguchi R, et al. Renal tubular dysgenesis. AfR 1991;157:383-4.

15 Guignard JP, Burgener F, Calame A. Persistent anuria in a neonate: a side effect of captopril? Int $\mathcal{f}$ Pediatr Nephrol 1981;2:133.

16 Duminy PD, Burger PD. Fetal abnormality associated with the use of captopril during pregnancy. $S$ Afr Med $\mathcal{F}$ 1981;60:805.

17 Rothberg AD, Lorenz R. Can capropril cause fetal and neonatal renal failure? Pediatr Pharmacol 1984;4:189-92.

18 Schubiger G, Flury G, Nussberger J. Enalapril for pregnancy-induced hypertension: acute renal failure in a neonate. Ann Intern Med 1988;108:215-16.

19 Patchett AA, Harris E, Tristram EW, et al. A new class of angiotensin converting enzyme inhibitors. Nature 1980 208:280-3.

20 Editorial. Are ACE inhibitors safe in pregnancy? Lancet 1989;ii:482-3.

21 Wolf G. Regulation of renal tubular cell growth: effects of angiotensin II. Exp Nephrol 1994;2:107-14.

22 Daemen MJ, Lombradi DM, Bosman FT, Schwartz SM. Angiotensin II induces smooth cell proliferation in the normal and injured rat arterial wall. Circ Res 1991;68:450-6.

$23 \mathrm{Re}$ R. Angiotensin and regulation of cellular growth. Pathophysiologic implications for cardiovascular and noncardiovascular tissues. Am f Hypertens 1991;4:21 7-19S.

24 Correa-Rotter R, Pérez-Castillo A, Chmielewski D, Rosenberg ME. Relationship between renin, angiotensinogen and histone $\mathrm{H} 2 \mathrm{~b}$ messenger ribonucleic acid in the maturing rat kidney. Nephron 1992;62:322-7.

25 Wolf G, Neilson EG. Angiotensin II induces cellular growth in cultured murine proximal tubular cells. Am $\mathcal{F}$ Physiol 1990;259:F768-77.

26 Wolf G, Kunco GS, Sun MJ, Neilson EG. Expression of homeobox genes in a proximal tubular cell line derived from adult mice. Kidney Int 1991;39:1027-33. 